\title{
UV properties of higher dimensional operators in Higgs Effective Field Theories from hidden symmetries
}

\section{Andrea Quadri*}

INFN, Sez. di Milano and Dip. di Fisica, Università degli Studi di Milano

via Celoria 16, I-20133 Milano, Italy

E-mail: andrea.quadri@mi.infn.it

\begin{abstract}
We present a systematic approach to the classification of the UV properties of higher dimensional operators spanned by $\Phi^{\dagger} \Phi$ (where $\Phi$ is the Higgs doublet) and ordinary derivatives thereof (e.g. $\left.\partial_{\mu}\left(\Phi^{\dagger} \Phi\right) \partial^{\mu}\left(\Phi^{\dagger} \Phi\right),\left(\Phi^{\dagger} \Phi\right)^{3},\left(\Phi^{\dagger} \Phi\right)^{4}, \ldots\right)$ in Higgs Effective Field Theories. The procedure is purely algebraic and thus regularization-independent. It relies on a novel set of hidden symmetries that can be formulated in an extended field space where the singlet $\Phi^{\dagger} \Phi$ is treated as a dynamical variable. The resulting relations stemming from such symmetries are valid to all orders in the loop expansion for off-shell 1-PI Green's functions (and not only for $S$-matrix elements). Oneloop applications are briefly discussed.
\end{abstract}

The European Physical Society Conference on High Energy Physics

5-12 July, 2017

Venice

${ }^{*}$ Speaker. 


\section{Introduction}

After the discovery at the LHC of a scalar particle whose properties are compatible with the Standard Model (SM) Higgs boson [1, 2] an important theoretical and experimental question is to constrain the potential triggering the electroweak spontaneous symmetry breaking (SSB). Effective Field Theories (EFTs) provide a useful tool to parameterize in a model-independent way the effects of Beyond-the-Standard-Model (BSM) physics induced by higher dimensional operators suppressed by inverse powers of a large energy scale $\Lambda$ [3]. In the EFTs approach one enumerates all possible operators compatible with the symmetries of the theory by organizing them in layers of a given dimension. When dealing with physical quantities like $S$-matrix elements, some ambiguities are present, associated with the equivalence of operators differing by terms proportional to the equations of motions. These ambiguities can be fixed by choosing a particular operator basis [4]. EFTs are of course not power-counting renormalizable. Their UV properties can be understood within renormalizability in the modern sense à la Weinberg and Gomis [5], namely all possible UV divergences can be reabsorbed provided that all possible operators compatible with the symmetries of the theory are introduced, at the price of adding higher and higher dimensional operators as the loop number increases. It has been recently realized by explicit one-loop computations that in the SM extended with dimension-6 operators a surprising pattern of cancellations exists, yielding a much more constrained set of UV divergences than one would expect on the basis of symmetry arguments only $[6,7,8]$. These cancellations have been traced back to the remnants of supersymmetry in a (non-supersymmetric) EFT $[9,10]$. A classification of one-loop non renormalization theorems for EFTs involving dimension six operators based on holomorphy arguments has been given in [11]. These results are valid at one-loop order for physical on-shell quantities since equations of motion are in general used in these computations. In [12] an alternative reformulation of the SSB sector of the SM has been proposed, based on the idea of using the singlet $\Phi^{\dagger} \Phi$ (after SSB) as the dynamical variable describing the physical scalar excitation. The advantage of this approach is the possibility to establish at one loop a diagrammatic separation between the SM and the BSM contributions to 1-PI Green's functions, which is in turn the consequence of some hidden functional symmetries.

The functional identities provide very strong constraints on the 1-PI Green's functions; these constraints are expressed in terms of 1-PI amplitudes involving insertions of a suitable set of external sources with a better UV behaviour than the quantized scalar fields. This in turn allows to use the techniques of Algebraic Renormalization $[13,14,15,16]$ in order to study the renormalization properties of the Higgs EFTs.

A unique deformation of the functional identities allows one to control the inclusion of the derivative operator $\partial_{\mu}\left(\Phi^{\dagger} \Phi\right) \partial^{\mu}\left(\Phi^{\dagger} \Phi\right)$ by modifying the 2-point function of the field describing the physical scalar excitation, while the interaction vertices are left unchanged.

\section{Functional Identities}

We denote by $\Phi$ the standard scalar field SU(2) doublet:

$$
\Phi=\frac{1}{\sqrt{2}}\left(\begin{array}{c}
i \phi_{1}+\phi_{2} \\
\phi_{0}+i \phi_{3}
\end{array}\right),
$$


where $\phi_{a}$ are the pseudo-Goldstone fields, $\phi_{0}$ the would be Higgs field and $v$ its expectation value. In order to use the combination $\Phi^{\dagger} \Phi$ (after SSB) as the dynamical variable associated with the singlet field $X_{2}$, describing the physical scalar mode, we need to introduce a Lagrange multiplier $X_{1}$ enforcing on-shell the condition

$$
X_{2}=\frac{1}{v}\left(\Phi^{\dagger} \Phi-\frac{v^{2}}{2}\right)=\sigma+\frac{1}{2 v} \sigma^{2}+\frac{1}{2 v} \phi_{a}^{2}
$$

The tree-level action of the model will be $S=S_{\mathrm{SM}}+S_{\mathrm{SSB}}$, where $S_{\mathrm{SM}}$ is the usual SM action involving the Yang-Mills, fermion, Yukawa, the gauge fixing and ghost terms and $S_{\mathrm{SSB}}$ replaces the SSB Higgs term and reads ${ }^{1}$

$$
\begin{aligned}
S_{\mathrm{SSB}}=\int d^{4} x & {\left[D_{\mu} \Phi^{\dagger} D^{\mu} \Phi-\frac{M^{2}}{2} X_{2}^{2}-\bar{c} \square c+\frac{1}{v}\left(X_{1}+X_{2}\right) \square\left(\Phi^{\dagger} \Phi-\frac{v^{2}}{2}-v X_{2}\right)\right.} \\
& \left.+\bar{c}^{*}\left(\Phi^{\dagger} \Phi-\frac{v^{2}}{2}-v X_{2}\right)+V\left(X_{2}\right)\right],
\end{aligned}
$$

with $D$ the usual covariant derivative and $V\left(X_{2}\right)$ a BSM potential in the $X_{2}$ field. $\bar{c}^{*}$ is an external source required to control the renormalization of the non-linear operator $\Phi^{\dagger} \Phi-\frac{v^{2}}{2}-v X_{2}$. In the present paper we consider the simplest case of a trilinear interaction $V\left(X_{2}\right)=g_{6} X_{2}^{3}$. Then one needs to add to Eq.(2.2) a further external source $R$ coupled to $X_{2}^{2}$.

The dependence on $X_{1}$ and $X_{2}$ is fully controlled by the following functional identities (a subscript denotes differentiation w.r.t. the variable):

$$
\Gamma_{X_{1}}=\frac{1}{v} \square \Gamma_{\bar{c}^{*}}, \quad \Gamma_{X_{2}}=\frac{1}{v} \square \Gamma_{\bar{c}^{*}}+3 g_{6} \Gamma_{R}-\square X_{1}-\left(\square+M^{2}\right) X_{2}-v \bar{c}^{*}+2 R X_{2} .
$$

By going on-shell with $X_{1}$ in Eq.(2.2) one recovers the classical SM action plus a dimension six operator $V\left(X_{2}\right)=g_{6} X_{2}^{3} \sim \frac{g_{6}}{v^{3}}\left(\Phi^{\dagger} \Phi-\frac{v^{2}}{2}\right)^{3}$. One should notice that Eqs.(2.3) still holds without any further external source if one adds a kinetic term $\int \frac{z}{2} \partial_{\mu} X_{2} \partial^{\mu} X_{2}$ for $X_{2}$ into Eq.(2.2), so that now the $X_{2}$-equation reads

$$
\Gamma_{X_{2}}=\frac{1}{v} \square \Gamma_{\bar{c}^{*}}+3 g_{6} \Gamma_{R}-\square X_{1}-\left((1+z) \square+M^{2}\right) X_{2}-v \bar{c}^{*}+2 R X_{2} .
$$

By going on-shell with $X_{1}$ this is equivalent to the inclusion into the action Eq.(2.2) of the dimension six operator

$$
\frac{z}{v^{2}} \partial^{\mu}\left(\Phi^{\dagger} \Phi\right) \partial_{\mu}\left(\Phi^{\dagger} \Phi\right)
$$

Eqs.(2.3) and (2.4) are valid to all orders in the loop expansion and for any (symmetric) regularization scheme. It should be noticed that at $z \neq 0$ the identities connecting amplitudes involving $X_{2}$-external legs with those without do not change with respect to the case $z=0$ (the $z$-dependent term in the right hand side of Eq.(2.4) being confined at tree-level). However the UV divergences of $X_{2}$-independent amplitudes change at $z \neq 0$. The reason is that the propagator $\Delta_{X X}$ of the combination $X=X_{1}+X_{2}$ now falls off for large momenta as $1 / p^{2}$ (instead as $1 / p^{4}$, as it happens at

\footnotetext{
${ }_{1}$ a further term $-\frac{m^{2}}{2 v^{2}}\left(\Phi^{\dagger} \Phi-\frac{v^{2}}{2}\right)^{2}$ is allowed in the above action [17] without altering the UV behaviour of the 1-PI amplitudes. Nevertheless the parameter $m$ drops out of physical amplitudes [17] and thus for the sake of simplicity it is set here to zero.
} 
$z=0)$ :

$$
\Delta_{X X}=\frac{i\left(-z p^{2}+M^{2}\right)}{p^{2}\left[(1+z) p^{2}-M^{2}\right]}
$$

Then the derivative interaction vertices in the first line of (2.2) become dangerous and spoil the milder UV behaviour of the $z=0$ theory. A complete classification of UV divergences at $z \neq 0$ is beyond the scope of the present work and is currently under investigation.

The action (2.2) is invariant under a U(1) BRST symmetry $s \bar{c}=S_{\bar{c}^{*}}, \quad s X_{1}=v x, \quad s c=0$ ensuring that the only physical scalar particle is described by the field $X_{2}$, while the combination $\sigma^{\prime}=\sigma-X_{1}-X_{2}$ cancels out together with $X_{1}$ to all orders in the loop expansion $[12,17]$.

\section{One-loop Effective Action}

The advantage of the reformulation of the SSB action in Eq.(2.2) is that it allows a one-loop diagrammatic decomposition of the SM and BSM-contributions of the complete 1-PI amplitudes (not only of on-shell quantities) and a classification of UV divergences in terms of Green's functions involving the external sources $R, \bar{c}^{*}$.

Indeed the BSM coupling $g_{6}$ only appear in the trilinear interaction vertex for $X_{2}$ and thus it can never contribute at one loop to amplitudes without external $X_{2}$-legs. On the other hand, amplitudes involving at least one external $X_{2}$-leg are uniquely fixed by Eq.(2.4) in terms of amplitudes that do not involve $X_{2}$ and which are thus $g_{6}$-independent.

In order to recover the 1-PI amplitudes in the standard formalism one needs to go on-shell with both $X_{1}$ and $X_{2}$. This amount to replace $X_{2}$ by the solution (2.1) to the $X_{1}$-equation of motion and by enforcing the tree-level $X_{2}$-equation of motion (at zero external sources)

$$
\square\left(X_{1}+X_{2}\right)=-\left(z \square+M^{2}\right) X_{2}+3 g_{6} X_{2}^{2},
$$

where $X_{2}$ is to be substituted by Eq.(2.1). Thus for example for any given amplitude involving $n$-insertions of the Higgs field $\sigma$ in the target theory (the one in the standard formalism), one has the following contributions:

- the pure SM part, equal to the 1-PI Green's function $\Gamma_{\sigma\left(x_{1}\right) \ldots \sigma\left(x_{n}\right)}^{(1)}$ in the $X_{1,2}$-theory;

- the BSM contribution, obtained by summing up all amplitudes with a $X_{1}$ or $X_{2}$-external leg that give rise upon the substitutions in Eqs.(2.1) and (3.1) to amplitudes with $n$-external $\sigma$ legs in the target theory.

The UV properties of the BSM contribution to these amplitudes are fixed by those of the kernels involving the external sources $R, \bar{c}^{*}$, which in turn can be easily classified. We refer the reader to [18] for a detailed application of this method and the complete classification of one-loop divergences of amplitudes involving the Higgs field in a SM Higgs EFT with an arbitrary derivative-independent potential $V\left(X_{2}\right)$. 


\section{Conclusions}

At one-loop level Higgs EFTs exhibit interesting unexpected cancellations of UV divergences that cannot be ascribed to gauge-invariance. We have presented an approach that allows for a complete classification of one-loop divergences in the presence of a derivative-independent SSB potential depending only on $\Phi^{\dagger} \Phi$. The main technical tool is the reformulation of the SSB mechanism at work in the SM by using the invariant $\Phi^{\dagger} \Phi$ as the new dynamical variable (after SSB). The correspondence with the standard formalism is achieved by going on-shell with $X_{1}$ and $X_{2}$. This in turn allows for a diagrammatic decomposition of the 1-PI amplitudes into a pure SM part and a BSM contribution, whose UV properties can be easily studied by classifying those of the kernels involving the external sources insertions. This provides a very effective way to compute the one-loop divergences of 1-PI amplitudes in the Higgs EFTs. We remark that the results hold for the full 1-PI amplitudes, without any restriction to on-shell physical quantitites as done before in the literature.

\section{References}

[1] S. Chatrchyan et al. [CMS Collaboration], Phys. Lett. B 716 (2012) 30 doi:10.1016/j.physletb.2012.08.021 [arXiv:1207.7235 [hep-ex]].

[2] G. Aad et al. [ATLAS Collaboration], Phys. Lett. B 716 (2012) 1 doi:10.1016/j.physletb.2012.08.020 [arXiv:1207.7214 [hep-ex]].

[3] See e.g. D. de Florian et al. [LHC Higgs Cross Section Working Group], doi:10.23731/CYRM-2017-002 arXiv:1610.07922 [hep-ph] for a recent summary.

[4] A summary of the most popular bases and their relation is given in A. Falkowski, B. Fuks, K. Mawatari, K. Mimasu, F. Riva and V. Sanz, Eur. Phys. J. C 75 (2015) no.12, 583 doi:10.1140/epjc/s10052-015-3806-x [arXiv:1508.05895 [hep-ph]].

[5] J. Gomis and S. Weinberg, Nucl. Phys. B 469, 473 (1996) doi:10.1016/0550-3213(96)00132-0 [hep-th/9510087].

[6] E. E. Jenkins, A. V. Manohar and M. Trott, JHEP 1310 (2013) 087 doi:10.1007/JHEP10(2013)087 [arXiv:1308.2627 [hep-ph]].

[7] E. E. Jenkins, A. V. Manohar and M. Trott, JHEP 1401 (2014) 035 doi:10.1007/JHEP01(2014)035 [arXiv:1310.4838 [hep-ph]].

[8] R. Alonso, E. E. Jenkins, A. V. Manohar and M. Trott, JHEP 1404 (2014) 159 doi:10.1007/JHEP04(2014)159 [arXiv:1312.2014 [hep-ph]].

[9] R. Alonso, E. E. Jenkins and A. V. Manohar, Phys. Lett. B 739 (2014) 95 doi:10.1016/j.physletb.2014.10.045 [arXiv:1409.0868 [hep-ph]].

[10] J. Elias-Miro, J. R. Espinosa and A. Pomarol, Phys. Lett. B 747 (2015) 272 doi:10.1016/j.physletb.2015.05.056 [arXiv:1412.7151 [hep-ph]].

[11] C. Cheung and C. H. Shen, Phys. Rev. Lett. 115 (2015) no.7, 071601 doi:10.1103/PhysRevLett.115.071601 [arXiv:1505.01844 [hep-ph]].

[12] A. Quadri, Int. J. Mod. Phys. A 32 (2017) no.16, 1750089 doi:10.1142/S0217751X17500890 [arXiv: 1610.00150 [hep-th]]. 
[13] O. Piguet and S. P. Sorella, Lect. Notes Phys. Monogr. 28 (1995) 1. doi:10.1007/978-3-540-49192-7

[14] R. Ferrari, P. A. Grassi and A. Quadri, Phys. Lett. B 472 (2000) 346 doi:10.1016/S0370-2693(99)01452-5 [hep-th/9905192].

[15] A. Quadri, JHEP 0304 (2003) 017 doi:10.1088/1126-6708/2003/04/017 [hep-th/0301211].

[16] A. Quadri, J. Phys. G 30 (2004) 677 doi:10.1088/0954-3899/30/5/009 [hep-th/0309133].

[17] A. Quadri, Phys. Rev. D 73 (2006) 065024 doi:10.1103/PhysRevD.73.065024 [hep-th/0601169].

[18] D. Binosi and A. Quadri, "Off-shell renormalization of dimension 6 operators in Higgs effective field theories," arXiv:1709.09937 [hep-th]. 